University of Nebraska - Lincoln

DigitalCommons@University of Nebraska - Lincoln

Is the magnetic anisotropy proportional to the orbital moment?

Ralph Skomski

University of Nebraska-Lincoln, rskomski2@unl.edu

Arti Kashyap

University of Nebraska-Lincoln, akashyap@Inmiit.ac.in

Axel Enders

University of Nebraska-Lincoln, a.enders@me.com

Follow this and additional works at: https://digitalcommons.unl.edu/physicsenders

Part of the Physics Commons

Skomski, Ralph; Kashyap, Arti; and Enders, Axel, "Is the magnetic anisotropy proportional to the orbital moment?" (2011). Axel Enders Publications. 26.

https://digitalcommons.unl.edu/physicsenders/26

This Article is brought to you for free and open access by the Research Papers in Physics and Astronomy at DigitalCommons@University of Nebraska - Lincoln. It has been accepted for inclusion in Axel Enders Publications by an authorized administrator of DigitalCommons@University of Nebraska - Lincoln. 


\title{
Is the magnetic anisotropy proportional to the orbital moment?
}

\author{
R. Skomski, ${ }^{\text {a) }}$ A. Kashyap, ${ }^{\text {b) }}$ and A. Enders \\ Department of Physics and Astronomy and Nebraska Center for Materials and Nanoscience, \\ University of Nebraska, Lincoln, Nebraska 68588, USA
}

(Presented 16 November 2010; received 25 September 2010; accepted 8 December 2010; published online 6 April 2011)

\begin{abstract}
The relation between orbital moment and magnetic anisotropy is investigated by model calculations, which show that only a part of the spin-orbit coupling contributes to the anisotropy. A large part of the anisotropy energy, about $50 \%$ for iron series elements and nearly $100 \%$ for rare-earths, is stored in the nonrelativistic part of the Hamiltonian. A feature important for x-ray magnetic circular dichroism is that the orbital moment of heavy atoms rotates with the spin moment, whereas in light atoms, the orbital moment is recreated in each different direction. In the discussion, we consider three examples of current interest in different areas of magnetism, namely, spin-orbit coupling in $\mathrm{Gd}^{3+}$ and $\mathrm{Eu}^{2+}$, surface anisotropy of $\mathrm{Nd}_{2} \mathrm{Fe}_{14} \mathrm{~B}$, and multiferroic magnetization switching using rare-earths. (C) 2011 American Institute of Physics. [doi:10.1063/1.3562445]
\end{abstract}

\section{INTRODUCTION}

It is well established that the spin-orbit coupling (SOC) is the origin of both orbital moment and anisotropy and that magnetocrystalline anisotropy requires an orbital moment. ${ }^{1}$ However, the relation between orbital moment and magnetic anisotropy has recently attracted renewed attention. Based on a widely quoted paper, ${ }^{2}$ it has become popular to equate the spinorbit energy with the magnetocrystalline anisotropy energy (MCA). It is correct that the SOC modifies the nonrelativistic Hamiltonian by a spin-orbit term, but the anisotropy energy is generally very different from the spin-orbit energy. For example, the $4 f$ shells in rare-earth magnets exhibit a rigid spin-orbit coupling, and the anisotropy energy is essentially equal to the crystal-field (CF) energy. ${ }^{3,4}$ This effect is exploited in high-performance permanent magnets such as $\mathrm{SmCo}_{5}$ and $\mathrm{Nd}_{2} \mathrm{Fe}_{14} \mathrm{~B} \cdot{ }^{3,4}$

The ratio of spin-orbit energy SOC energy to crystalfield or band energy has far-reaching implications, from the physical understanding of orbital-moment quenching to the interpretation of experimental methods such as x-ray magnetic circular dichroism (XMCD). For example, the orbitalmoment contribution to an XMCD signal may involve two different mechanisms, namely, the rotation of the orbital moment and its collapse. A transparent real-space interpretation is in terms of orbital currents, ${ }^{5}$ which are particularly strong in magnets with rotational symmetry, such as monatomic transition-metal wires. Intriguingly, the rigid rareearth spin-orbit coupling (large SOC) has recently been questioned for $4 f^{7}$ ions $\left(\mathrm{Gd}^{3+}\right.$ and especially $\left.\mathrm{Eu}^{2+}\right){ }^{6}$

\section{CALCULATIONS AND RESULTS}

To investigate the spin-orbit and crystal-field contribution to the magnetic anisotropy, we use the quasiclassical model Hamiltonian

\footnotetext{
${ }^{\text {a) }}$ Author to whom correspondence should be addressed. Electronic mail: rskimski@neb.rr.com.

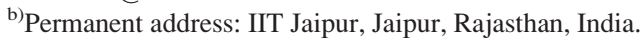

$$
E=-\lambda \mathbf{S} \cdot \mathbf{L}+A_{\circ}\left(L_{\mathrm{x}}^{2}+L_{\mathrm{y}}^{2}+L_{\mathrm{z}}^{2}\right)-A\left(L_{\mathrm{z}}^{2}-\frac{1}{2}\left(L_{\mathrm{x}}^{2}+L_{\mathrm{y}}^{2}\right)\right)
$$

where $\lambda$ is the spin-orbit coupling constant. The effective crystal- or ligand-field parameters $A>0$ and $A_{\mathrm{o}}>A$ describe the nonrelativistic electronic structure of the magnet. Note that hybridization (band structure) and electrostatic crystal fields have very similar effects on orbital moment and anisotropy, in molecules known as ligand-field interaction. ${ }^{5,7}$ In the present model, $0 \leq L^{2} \leq 1$, corresponding to fully quenched $\left(L^{2}=0\right)$, partially quenched $\left(0<L^{2}<1\right)$, and unquenched $\left(L^{2}=1\right)$ orbital moments. For simplicity, we also assume that $S^{2}=1$. Other choices of $L$ and $S$ do not yield additional physics.

The parameter $A_{\mathrm{o}}$ describes the quenching of the orbital moment due to the crystal field. Physically, the $d$ and $f$ electrons prefer eigenstates whose overlap with neighboring electron clouds are small. The creation of an orbital moment corresponds to a local electron current which partially enters otherwise avoided regions and therefore costs CF energy. ${ }^{4,5}$ Both $A$ and $A_{\mathrm{o}}$ may exceed $1 \mathrm{eV}$, especially in transitionmetal magnets, and the condition $A_{\mathrm{o}}>A$ ensures that there are no orbital moments in the absence of SOC. The case $A_{\mathrm{o}}=A$ is approximately realized in free-standing monatomic wires, where a small SOC gives rise to a huge orbital moment. ${ }^{4,8}$ Bringing such a wire into contact with a substrate destroys the orbital moment and effectively reinstates $A_{\mathrm{o}}>A$.

For small and moderate spin-orbit coupling $\left(L^{2}<1\right)$, minimization of Eq. (1) with respect to $L_{\mathrm{z}}$ and $L_{\mathrm{x}}$ yields

$$
\begin{gathered}
\lambda S_{\mathrm{z}}=2\left(A_{\mathrm{o}}-A\right) L_{\mathrm{z}} \\
\lambda S_{\mathrm{x}}=2\left(A_{\mathrm{o}}+A / 2\right) L_{\mathrm{x}} .
\end{gathered}
$$

Due to the tensor character of the orbital term in Eq. (1), $\mathbf{S}$ and $\mathbf{L}$ are generally noncollinear, but there is no mixing if $\mathbf{S}$ is parallel to any of the magnet's principal axes. For spin orientations in the $z$ and $x$ directions, the orbital moments are 


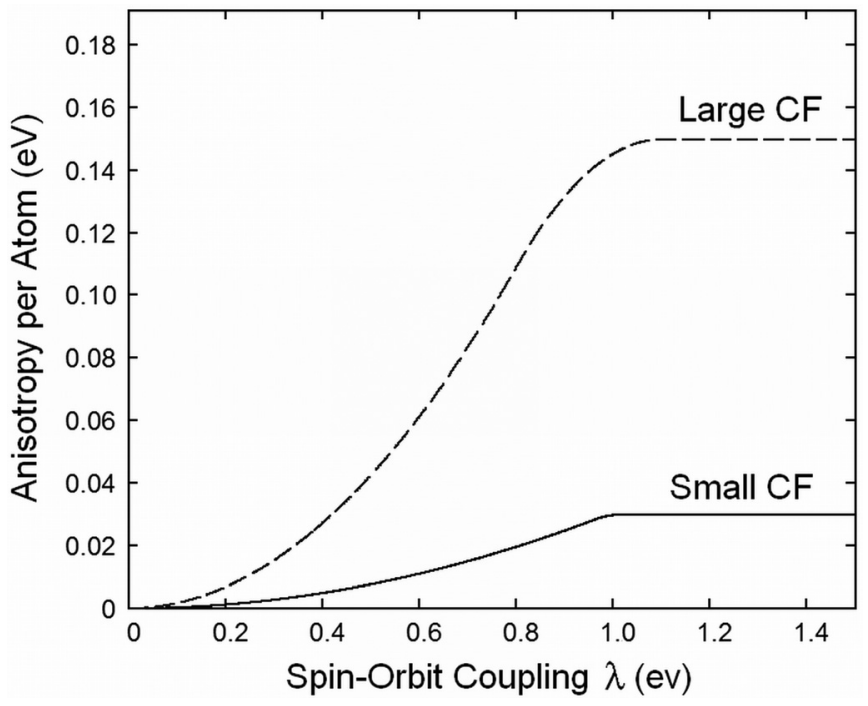

FIG. 1. Anisotropy per atom as a function of the spin-orbit coupling (SOC) strength for two different crystal fields $(\mathrm{CF})$. Iron-series atoms have large $\mathrm{CF}$ but small SOC, whereas rare-earths have small CF due to screening but large SOC. For the palladium and platinum series, the ratio of the two parameters is intermediate.

$[1 / 2] \lambda /\left(A_{\mathrm{o}}-A\right)$ and $[1 / 2] \lambda /\left(A_{\mathrm{o}}+A / 2\right)$, respectively. The anisotropy energy is obtained by substituting the orbital moments into Eq. (1) and comparing the total energies for the two directions:

$$
E_{a}=\frac{\lambda^{2}}{4}\left(\frac{1}{A_{\circ}-A}-\frac{1}{A_{\circ}-A / 2}\right) .
$$

The corresponding first uniaxial anisotropy constant $K_{1}=E_{\mathrm{a}} / V_{\mathrm{at}}$, where $V_{\text {at }}$ is the volume per atom. Equation (3) is of the familiar type $K_{n} \sim \lambda^{2 n} / \Delta A^{2 n-1}$, where $n$ is the order of the anisotropy. This result was first obtained by Bloch and Gentile ${ }^{1}$ for cubic anisotropy $(n=4)$.

By substituting $L_{\mathrm{z}}$ and $L_{\mathrm{x}}$ into Eq. (1) we can separately evaluate the SOC energy, $E_{\mathrm{SOC}}=2 E_{\mathrm{a}}$. In other words, only $50 \%$ of the spin-orbit energy translates into anisotropy energy. The other $50 \%$ are used for the increase in crystal-field energy, which accompanies the formation of the orbital moment.

Minimization of Eq. (1) and taking into account that $L^{2} \leq 1$ yields a number of limiting and intermediate scenarios. Figure 1 shows two anisotropy energies as a function of the spin-orbit constant $\lambda$. For small $\lambda$, the behavior is quadratic, as in Eq. (3). For large $\lambda$, the anisotropy reaches a plateau at $E_{\mathrm{a}}=3 A / 2$. The two limits corresponds to the well-known cases of $3 \mathrm{~d}$ (iron-series) and $4 f$ (lanthanide) anisotropies, respectively. Figure 2 shows the anisotropy energy relative to several spinorbit energy terms and illustrates that there is no simple relation between spin-orbit coupling and anisotropy energy.

\section{DISCUSSION}

Let us discuss three examples of importance in different areas of magnetism, namely, the spin-orbit coupling in halffilled $4 f$ shells, the surface anisotropy of $\mathrm{Nd}_{2} \mathrm{Fe}_{14} \mathrm{~B}$, and the exploitation of the SOC in multiferroic switching without strain.

A recent paper has questioned the applicability of the rigid-coupling approach to $4 f^{7}$ ions, by arguing that $L=0$

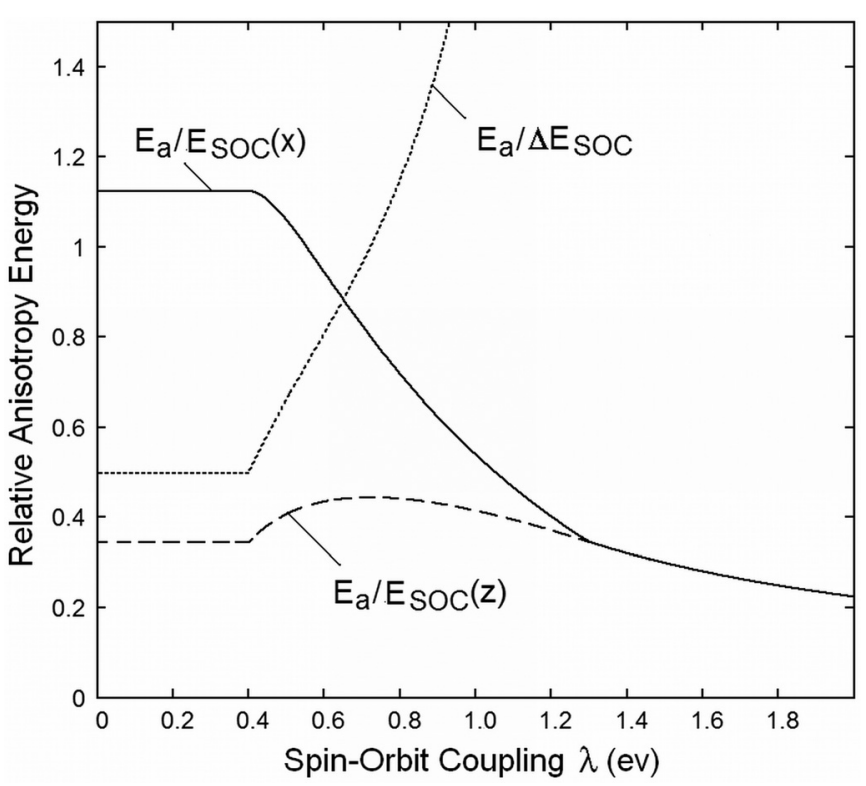

FIG. 2. Ratios of anisotropy energy $E_{\mathrm{a}}$ and spin-orbit coupling SOC for different directions. Reference 2 corresponds to a horizontal line with a ratio of one.

for ions such as $\mathrm{Gd}^{3+}$ and $\mathrm{Eu}^{2+}$, so that $L$ and $S$ decouple. ${ }^{6}$ However, the total $L$ is only one consideration. Both quenched wave functions, $\cos (m \phi)$ and $\sin (m \phi)$, and quenched wave functions

$$
\left|\Psi_{ \pm}(m)>=\right| \Psi_{\cos }(m)> \pm \mathrm{i} \mid \Psi_{\sin }(m)>
$$

yield $L=0$ for Gd (Fig. 3), but this does not mean that the orbital moments decouple from the spin moment. The Hund'srules ground state of $\mathrm{Gd}^{3+}$ has seven $\uparrow$ electrons, so that all available orbital states $(m=-3,-2,-1,0,1,2,3)$ are occupied and individual electrons cannot interact with the crystal field. This argument also applies to the possible partial quenching of one-electron $4 f$ states caused by intra-atomic Coulomb interactions. If the orbital degrees of freedom $( \pm m)$ were decoupled from the spin, they would yield, for example, a contribution to the heat capacity. However, Eu spectroscopy does not support such low-lying excitations, and the lowestlying excited term $(S=5 / 2)$ has an excitation energy of about $4.0 \mathrm{eV},{ }^{9}$ as compared to typical $\mathrm{CF}$ energies of order $20 \mathrm{meV}$. Reference 6 contains the interesting argument that $\mathrm{Eu}^{2+}$ crystal fields tend to be larger than $\mathrm{Gd}^{3+}$ crystal fields. However, this does not invalidate the involvement of terms with $S=5 / 2$, that is, terms with nonzero $L$ and with net spin-orbit coupling

A practically important feature in rare-earth permanent magnetism is the behavior of neodymium in $\mathrm{Nd}_{2} \mathrm{Fe}_{14} \mathrm{~B}$, whose anisotropy is reduced at the (001) surface. The

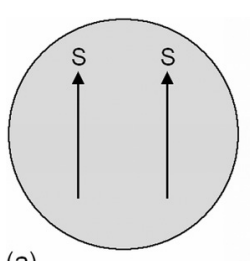

(a)

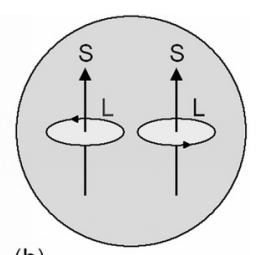

(b)
FIG. 3. One-electron character of a Gd $4 f$ shell: (a) quenched and (b) unquenched. In both cases, the total $L$ equals zero. 


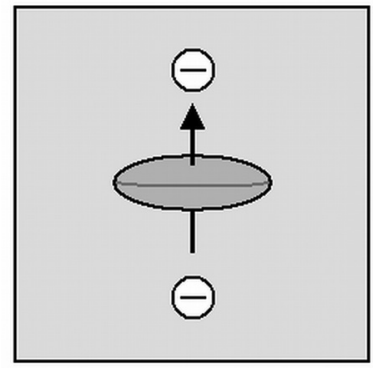

(a)

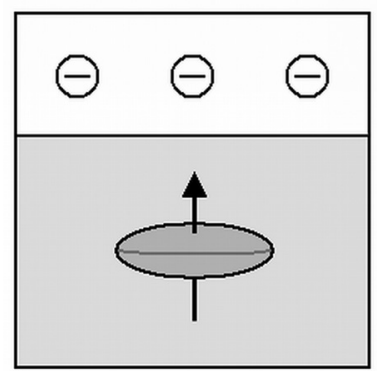

(c)

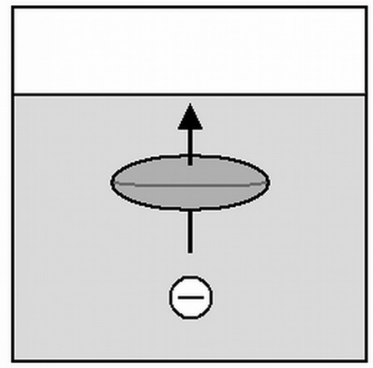

(b)

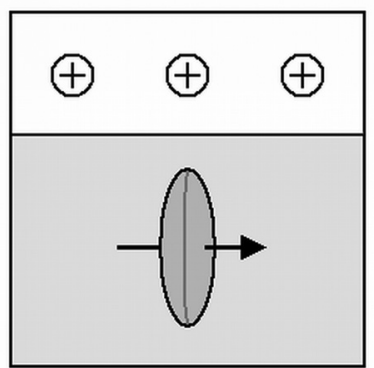

(d)
FIG. 4. Crystal-field of an oblate $4 f$ shell $\left(\mathrm{Nd}^{3+}\right)$ : (a) schematic neighborhood in bulk $\mathrm{Nd}_{2} \mathrm{Fe}_{14} \mathrm{~B}$, (b) (001) surface in $\mathrm{Nd}_{2} \mathrm{Fe}_{14} \mathrm{~B}$, and (c)-(d) multiferroic switching (schematic).

corresponding loss in coercivity is typically compensated by the use of dysprosium, but element-strategic consideration discourage the use of Dy, and current experimental and theoretical work is tackling this problem. ${ }^{11,12}$ Figures 4(a)-4(b) shows how the reduced surface anisotropy can be explained by the CF interaction of the $\mathrm{Nd}$ atoms at the surface. $\mathrm{Nd}^{3+}$ has a negative Stevens coefficient (oblate $4 f$ shell), so that the orientation of the neighboring crystal-field charges in bulk $\mathrm{Nd}_{2} \mathrm{Fe}_{14} \mathrm{~B}$ (positive crystal-field coefficient $A_{2}{ }^{0}$ ) yield easy-axis anisotropy. The effect is weakened at the (001) surface, because a negative crystal-field charge is removed from the top, Fig. 4(b). The resulting surface anisotropy then modifies the coercivity. ${ }^{13}$

Multiferroic materials have recently attracted much attention, for example, in spin electronics. Traditional systems exploit magnetoelastic strain, but the switching of the preferential magnetization direction can also be realized by crystal-field interactions. ${ }^{5}$ Precursors of this mechanism are easy-axis changes in $\mathrm{Sm}_{2} \mathrm{Fe}_{17} \mathrm{~N}_{\mathrm{x}}$ due to interstitial nitrogen $^{14,15}$ and changes of the magnetization of $\mathrm{Ni} / \mathrm{Cu}(001)$ thin films due to adsorbed hydrogen. ${ }^{16}$ Figures 4(c)-4(d) illustrate the switching mechanism, which is triggered by the change in the electric dipole moment of the ferroelectric. In lowest order, the magnetization direction is given by the electrostatic quadrupole interaction of the rare-earth $4 f$ shells with the crystal-field gradient. ${ }^{3,4}$ Since the crystal-field source is the ferroelectric layer, the present interaction is proportional to electrostatic dipole-field gradient and therefore decreases as $1 / D^{4}$ with increasing distance $D$ between $4 f$ ion and electrostatic dipole. By comparison, uniaxial anisotropies exhibit a $1 / D^{3}$ dependence.

\section{CONCLUSIONS}

In summary, we have investigated the intriguing interplay between spin-orbit and crystal-field interactions in ferromagnets. Magnetization rotations in a crystal change both the spin-orbit and crystal-field energies, and only a part of the spin-orbit energy translates into anisotropy, namely about $50 \%$ for itinerant magnets and about $1 \%$ for rare-earth magnets. The latter mechanism allows an intuitive description of effects such as surface anisotropies in rare-earth permanent magnets and multiferroic switching of rare-earth spins due to electric fields. The case of $4 f^{7}$ ions is less transparent, although we suggest that the vanishing orbital moment is unrelated to the one-electron quenching of the iron-series orbital moments.

\section{ACKNOWLEDGMENTS}

This research is supported by NSF MRSEC, ARPA-E, BREM, DST, DOE, and NCMN.

${ }^{1}$ F. Bloch and G. Gentile, Z. Phys. 70, 395 (1931).

${ }^{2}$ P. Bruno, Phys. Rev. B 39, 865 (1989).

${ }^{3}$ J. F. Herbst, Rev. Mod. Phys. 63, 819 (1991).

${ }^{4}$ R. Skomski and J. M. D. Coey, Permanent Magnetism (Institute of Physics, Bristol, 1999).

${ }^{5}$ R. Skomski, Simple Models of Magnetism (University Press, Oxford, 2008).

${ }^{6}$ G. van der Laan, E. Arenholz, A. Schmehl, and D. G. Schlom, Phys. Rev. Lett. 100, 067403 (2008).

${ }^{7}$ C. J. Ballhausen, Ligand Field Theory (McGraw-Hill, New York, 1962).

${ }^{8}$ M. Komelj, C. Ederer, J. W. Davenport, and M. Fähnle, Phys. Rev. B 66, 140407 (2002).

${ }^{9}$ K. N. R. Taylor and M. I. Darby, Physics of Rare Earth Solids (Chapman and Hall, London, 1972).

${ }^{10}$ D. Schmitt, J. Phys. F 9, 1745 (1979).

${ }^{11}$ S. Sugimoto, Proc. REPM'10, Eds. S. Kobe and P. McGuinness, Jozef Stefan Institute, Ljubljana2010, p. 103.

${ }^{12}$ H. Moriya, H. Tsuchiura, and A. Sakuma, Proc. REPM'10, edited by S. Kobe and P. McGuinness (Jozef Stefan Institute, Ljubljana, 2010), p. 154.

${ }^{13}$ R. Skomski, X.-H. Wei, and D. J. Sellmyer, IEEE Trans. Magn. 43(6), 2890 (2007).

${ }^{14}$ J. M. D. Coey and H. Sun, J. Magn. Magn. Mater. 87, L251 (1990).

${ }^{15}$ R. Skomski and J. M. D. Coey, J. Appl. Phys. 73, 7602 (1993).

${ }^{16}$ D. Sander, W. Pan, S. Ouazi, J. Kirschner, W. Meyer, M. Krause, S. Müller, L. Hammer, and K. Heinz, Phys. Rev. Lett. 93, 247203 (2004). 